IX.

Aus der Staats-lrrenanstalt zu Lübeck (Dirig. Arzt Dr. O. Wattenberg).

\title{
Ehescheidung bei inducirtem Irresein
}

an einem Gutachten erläutert

von.

Dr. Ernst Kalmus,

Assistenzarzt.

$\mathrm{N}_{\text {achstehende Mittheilung leitet ihr Recht auf Interesse vornehmlich }}$ daraus her, dass die Folie à deux hierin zum ersten Mal -- vielleicht überhaupt, sicherlich aber in der Literatur - den Gegenstand gutachtlicher Aeusserung im Hinblick auf den § 1569 des Bürgerlichen Gesetzbuches bildet. Ehescheidung bei communicirtem Irresein scheint bei unbefangener Beurtheilung beinahe als selbstverständlich, ja fast als ideal für das Zutreffen der natürlichen Vorbedingungen, weil, schon rein klinisch genommen, die innige Ehegemeinschaft als solche den ausschlaggebenden, schädlichen Factor darstellt. Doch Erwägungen des gesunden Menschenverstandes und strenge Eingliederung in einen Gesetzesparagraphen sind zweierlei. Schwierigkeiten derart sind dem psychiatrischen Gutachter bekannt; es sei nur an den unglücklich eng gefassten $\S 51$ des Strafgesetzbuches erinnert. Um unvorhergesehene Spielarten pathologischer Erscheinungen in festgefügte juristische Begriffe einzuordnen, sieht man sich leicht, wenn auch ungern, gelegentlich veranlasst, den widerstrebenden Einzelfall, dem gewünschten Endzweck zu Liebe, in das Procrustesbett des Gesetzes zu zwängen.

Indessen, statt dem Falle Gewalt anzuthun, wird man es, wenn irgend möglich, vorziehen dem Gesetze eine neue, erweiterte Auslegung zu Theil werden zu lassen.

Der Ehescheidungsparagraph scheint nach der bisherigen, allerdings kurzen Erfahrung in seiner Fassung glücklich formulirt zu sein. Er 
lässt der psychiatrischen Entscheidung weitgehende Freiheites. Kein Fall ist bekannt geworden, bei dem die irrenärztliche Auffassung, nicht aber der Wortlaut des Gesetzes eine Scheidung wegen Geisteskrankheit zuliess. Allerdings hat man sich ärztlicherseits überall, iu den theoretischen Erörterungen sowohl wie in der Praxis, peinlicher Zurückhaltung und sorgsamer Kritik befleissigt; man hat die sociale Bedeutnng der Ehe nach strengsten Grundsätzen in Rechnung gezogen, damit aus der Wohlthat des Gesetzes keine Plage werde.

Man hat sich im Allgemeinen den bekannten juristischen Erläuterungen Len el's') angeschlossen. Diese haben sich, soweit ersichtlich, bisher als durchgehend nützlich und zutreffend erwiesen. Für unseren Fall siud sie unzureichend. Weder das Bewusstsein der geistigen Gemeinschaft fehlt unserer Kranken noch der Wille, dieselbe zu pflegen; auch die moralischen Fähigkeiten sind in hinreichendem Maasse crhalten. Die materielle Möglichkeit eines verständnissvollen Beisam menlebens ist gegeben, und trotzdem die Ehescheidung indicirt, weil oine Wiedervereinigung den heute gesunden Ehegatten der sicheren Gefahr des inducirten Irreseins aussetzen würde. Also Verhältnisse ganz anderer Art, als die in den bisherigen Definitionen vorausgesetzten, kommen zur Geltung; neue Gesichtspunkte eröffnen sich. Der Fall gewinnt principielle Bedeutung, weil er die Frage löst, ob generell bei inducirtem Irresein zwischen Ehegatten die gesetzliche Scheidung gerechtfertigt ist.

Der Fall hat ferner als ein sogenannter "reiner" klinisch-casuistische Bedeutung. Bevor wir daran einige Bemerkungen knüpfen, sei Krankengeschichte und Gutachten mitgetheilt.

Ich statte an dieser Stelle meinem hochverehrten Chef, Herrn Dr. Wattenberg, für freundliche Ueberlassung des. Falles und gütigen Rath ergebenen Dank ab.

\section{Vorgeschichte.}

Frau M., geboren 185T, zu C., evangelisch, stammt von einem Vater, der Maler war, als Trinker bezeichnet wird und an Wassersucht verstorben ist, von einer Mutter, die von Anverwandten als "roh" und "herrschsüchtig, ähnlich der Tochter" geschildert wird und in der Jugend einmal voräbergehend erblindet war. Ein Bruder und eine Schwester sind gesund. Als Kind machte sie Masern durch, besuchte die Volksschule und erlernte die Näherei. Sie nahm eine Stelle als Wirthschafterin an, bei einem alten Herrn, der sie jedoch bald, nachdem siu zu ihm in ein intimes Verhältniss getreten war, wieder entliess. Dann kam sie in gleicher Eigenschaft zu einem Major in $X$ und knüpfte

1) S. Bericht der Jahresvers. d. Ver. der deutschen Irrenärzte. 1900. 
auch mit diesem Geschlechtsverkehr an. Die Folge war ein kind, welches starb. Gleichzeitig soll sie mit zahlreichen anderen Männern, angeblich elf, Ungang gehabt haben. Später lram sie als Wirthschafterin zu einem Herrn, den sie nach und nach röllig ausgesogen haben soll. Für die folgenden Jahre ist ihr Verbleib nicht sicher festzustellen. Sie hielt sich an verschiedenen. Orten auf, u. a. in H., wo sie ein zweites Kind geboren haben soll, schliesslich in G., wo sie mit einem Handelsmann im Concubinat lebte und ihren unsittlichen Lebenswandel in so aufälliger Weise fortsetzte, dass die Polizei auf sie aufmerksam wurde und sie 1880 aus G. verwies.

Einige Jahre darauf trat sie bei ihrem jetzigen Mann als Haushälterin eir. Er war Wittwer und hatteaus erster Ehe, die sehr glücklich war, 2 Kinder. Schon nach 14 Tagen kündigte er ihr wegen Misshandlung dieser Kinder. Trotzdem bliob sio. M. liess sie gewähren, erwies sich ihr gegenüber schwach und widerm standslos, ja verlobte sich mit ihr nach weiteren 14 Tagen. Das eheliche Verhältniss war schlecht. Eine Schwägerin und der Schwiegersohn berichten übereinstimmend und unabhängig von einander: Schon am Hochzeitstage kam es zu einer lärmenden Scene. Die Frau führte die Herrschaft im Hause, tyrannisirte tie Familie, behandelte den willensschwachen Mann wie ein unnündiges Kind, verwaltete das Geld, erbrach den Secretär, wenn es ihr kurze Zeit vorenthalten wurde, oder nahm es dem Manne Nachts aus der Tasche. Sie liess ihn oft hungern, so dass er sich mehr als einmal gezwungen sah, Nachts hinunterzuschleichen und sich trockenes Brod aus dem Essschrank zu holen. Ja, sie prügelte ihn sowohl wie die Kinder aus erster Ehe. Eine Tochter soll in Folge dessen das Gehör verloren haben; über die Misshandlungen liegen ärztliche Atteste ror. Endlich wird mitgetheilt, dass sie.während der Ehe noch Besuche ihres früheren Liebhabers, des Majors, empfing, mit ihm correspondirte und Geschenke entgegennahm, letzteres mit Wissen des Ehemannes.

Der Ehe entstammen ein lebender Sohn, zwei jung verstorbene Kinder. and eine Fehlgeburt.

Nach 12jähriger Ehe wurde Frau M. am 14. Aprì 1896 in die hiesige Irrenanstalt aufgenommen. Seit einem halben Jahre befand sich der Ehemann ebendaselbst in Behandlung. Während dieser jedoch am 1. November 97 als geheilt entlassen wurde, ist die Frau bis heute in der Anstalt verblieben.

Die Geisteskrankheit der Frau, üher deren erste Anfänge nichts beliannt ist, entwickelte sich anscheinend schleichend. Der Einzige, der darüber hätte Auskunft geben können, wax der Mann, dessen Urtheil aber wegen der eigenen psychischen Störung ohne Werth war. Die Schwierigkeiten für die ärztliche Beurtheilung steigerten sich dadurch, dass die Wahnvorstellungen und lrankhaften Handlungen beider Ehegatten sich zu einem anfangs unentwirrbaren Knäuel mit einander verflochten. Da aber gerade diese gegenseitigen Beziehungen beider Krankheitsfalle für unseren Zweck von ausscllaggebender Bedeutung sind, ist eine ausführlichere Darstellung der Vorgeschichte erforderlich. 
Wir geben zunächst einen Auszug aus den Polizei- und Staatsanwaltschafts-Acten der Ehefrau:

Frau M. stand Ende 1895 vor dem hiesigen Schöffengericht unter der Anklage, durch Iautes Schimpfen auf öffentlicher Strasse groben Unfug verübt zu haben, einen Vorgesetzten und einen Collegen ihres Mannes durch beleidigende Zurufe ("Ehrabschneider", "Eheschänder") und körperliche Misshandlung insultirt zu haben. Sie wurde wegen Geisteskrankheit freigesprochen. Der Sachverständige, Herr Physicus Dr. Riedel, stellte „chronische Verrüoktheit (Paranoia)" fest. Sie leide an Sinnestäuschungen und Verfolgungswahn. In dem Gutachten heisst es:

„Sie glaubte von den Passanten auf der Strasse, vamentlich von den Jebrern anzügliche Redensarten zu hören, sie sab die Passanten ausspucken und Geberden machen, sie hörte aus dem Hause des Schulrathes "Pudel"* rufen u. dergl. mehr. ... . Frau M. ist der festen Ueberzeugung, dass ihr Mann bereits seit 16 Jahren, seit seiner ersten Anstellung ungerecht verfolgt wird und dass noch dauernd seitens aller Behörden gegen ihren Mann und sie selbst agitirt wird . . . "4.

Wegen fortgesetztel Collisionen mit der Aassenwelt wurde sie am 7. April 96 zwecks Ueberführung in die Irrenanstalt entmündigt. In dem diẻsbezüglichen Physikats-Gutachten, dem zufolge "eine Heilung in absehbarer Zeit nicht zu erwarten sei", sind wörtliche Auslassungen der Kranken mitgetheilt, die das Verhältniss zum Manne betreffen und daher hier von Interesse sind:

„Diese Zurufe (Sinnestäuschungen) erfolgen auf Anstiften der Behörde, welche auch früher die Leute beauftragt, zu sagen, mein Mann sei verrückt und stinke. Dass mein Mann gesund ist, ist durch ärztliches Attest erwiesen. Sagen Sie mir doch, was er eigentlich für eine Schuld begangen hat, dass man ihn so verfolgt. Ich werde so lange dagegen angehen, als ich athmen kann, und sollte ich mein Leben hingeben. Ich werde nicht ruhen, bis die Behörde das. untersucht hat und sollte man mich hinter die Mauern der Irrenanstalt bringen. Mein Mann ist überhaupt nur darum in den Staatsdienst gewählt worden, um ihn verrückt zu machen. Die Polizeibehörde hat seiner Zeit Befehl gegeben, die Sachen auf dem Boden zu zerschneiden, um meinen Mann daraufhin entmündigen zu können. Seit 12 Jahren werde ich ungerecht von den Gerichten behandelt, so z. B. vor zwei Jahren gelegentlich einer Differenz mit einem 'Töpfer, welcher den Ofen nicht in der versprochenen Weise resetzt hatte. Der Töpfer schwur einen Meineid, so dass ich 100 Mark zahlen musste".

Das Gutachten fährt fort: „Was das Verhältniss zu den Miethern im Erdgeschoss betrifft, in welchen Frau M., wie in allen, die die Wohnung besichtigt haben, Angestiftete der Behörde erblickte, so behauptet Frau M., dass dieselben Nachts zur Zeit von 10 bis 1 Uhr einen unglaublichen Lärm ausführten, sie schlügen mit Deckeln, Thüren und Instrumenten, man höre Schüsse, Bellen und Stimmen. Sie und ihr Sohn arbeiteten denn auch gegen den Lärm an. Unzweifelhaft handelt es sich hierbei um Gehörshallucinationen bei Frau M., vielleicht auch bei dem Sohn, welcher auch angiebt, 
dass er, wenn er allein aufder Strasse gehe, von anderen Jungen ",P'Pdel.6. und "'Studio" $"$ gerufen werde".

\section{Status.}

Sie ist von kräftigem Körperbau, gedrungen, breitschulterig, in gutem Ernährungszustande. Das Gesicht ist massig, von fahler Farbe, die Züge sind markirt, Stirn steil und riedrig, Nase nach oben geschweift. Die Ohren sind ziemlich klein und zeigen geringe Abweichungen von der Norm. Lippen schmal und zusammengekniffen. Der rechte Mundwinkel ist weniger gut innervirt. Andere körpẹlliche Störungen bestehen nicht.

Der Geisteszustand der Frau M. hat, wie vorweg bemerkt werden sall, während des über 6jährigen Anstaltsaufenthalts eine principielle Aenderung nicht erfabren. Von einer chronologischen Darstellung ihres Verhaltens kann daher Abstand genommen werden, um so mehr, als sich das Krankheitsbild erst bei rückschauender Gesammtbeurtheilung in vollem Lichte darstellt.

Die Kranke war vom ersten Tage an mürrisch und unzugänglich; sie hat es stets abgelehnt; über ihre Gedankenwelt im Zusammenhange Bericht zu erstatten, und beharrt ärztlichen Fragen gegenüber, besonders seit den letzten Jahren, in Schweigen. Unsere Schlüsse gründen sich auf gelegentliche, aber nicht allzuseltene, im Affect gethane Aeusserungen, auf neuerliche Mittheilungen der Kranken an das Pflegepersonal, auf schriftliche Ergüsse, eine Reihe keiner Einzelzüge, auf Benehmen, Lehensgewohnheiten und Handlangen.

Der Gesichtsausdruck ist finster und trotzig: der Blick verräth Fass, Misstrauen und Verbissenheit. Sie lebt einsam auf ihrem Zimmer, sitzt müssig am Fenster oder liegt halbangekleidet anf ihrem Bette. Zeitweise ist sie mit Lesen oder Handarbeiten beschäftigt. Oft bleibt sie wochenlang im Bett und hält die Stirn mit feuchten Handtüchern bedeckt. Honatelang verlässt sie das Zimmer nicht. Sie ist unordentlich und wenig sauber in ihrer Kleidung, trägt ein Pack alter, werthloser Papiere und Gebrauchsgegenstände in einem Korbe bei sich, bewahrt viele Wochen Speisen in ihrer Schublade auf. Ein Eingriff in ihre sonderbaren Gewohnheiten löst zornige Erregung aus.

Bei guter Laune unterhält sie sich gelegentlich mit Pflegerinnen und Nitkranken, ausnahmsweise bei körperlicher Indisposition auch mit dem Arzt. thre Rede ist klar und geordnet. Oft klagt sie überKopfschmerz und schlechten Schlaf, beginnt dann regelmässig mit Beschwerden über das unzureichende und ungeniessbare Essen und erzählt in erbitterten Worten ron dem ihr angeblich zugefügten Unrecht und den an ihr begangenen Verbrechen. Geselligen Anschiuss oder gar Preundschaft hat sie nie gesucht, anch dort nicht, wo ein geneigtes Obr für ihre Mittheilungen zu finden war. Auf solehe Kranke suchte sie geflissentlich einzuwirken und durch geschickte Darsteling ihrer Lebensschicksale die Realität ihrer wahnhaften Erlebnisse zu erweisen. Mehr als einmal gelang es, ihren verderblichen Einfluss auf andere Kranke festzustellen, sodass eine räumliche Trennung nothwendig wurde, die dann auch von Erfolg begleitet war.

Ein für uns lehrreiches Beispiel aus jüngster Zeit sei kurz erwäbnt: 
Die 33jährige Frau E., welche ełenfalls an einer Geisteslirankheit mit Sinnestäuschungen leidet, wurde 1/2 Jahr nach der Aufnahme in die Anstalt aus anderen Gründen auf diejenige Abtheilung verlegt, die Frau M. bewohnt. Sie trat mit dieser in Berührung und bald wurde eine Verschlechternng ihres Befindens bemerkbar. Bẹi Frau E. griffen neue, bisher nie zu Tage getretene Wahnvorstellungen Platz; nie vorher erhobene Anschuldigungen gegen die Aerzte in der Anstalt wurden verlautbar; das Benehmen der Kranken und die Ausdrucksform ihrer Psychose hatte eine durchgreifende Aenderung and Ausgestaltung erfahren und zwar in getreuer Anlehnung an das Wahnsystem der Frau M. Auch Frau E. erklärte von nun an Oberin und Pflegerinnen als „Bestien", die „die Huren“ der beiden Aerzte wären. "Die beiden Schweine sorgen dafür, dass das Aaszeug hier im Hause frei ein- and ausgehe und die beste Kost habe. Uns anständigen Weibern giebt man Gift auf das Brod und zwischen das Essen, damit wir bei Seite geschafft werden. Das Volk wird von der Staatsanwaltschaft verfolgt und hier spielt sich die Bande auf. Mein Mann hat mich sicher hier nicht herbringen lassen; das haben andere beruntergekommene Subjecte gethan. $\mathrm{Na}$, ich schwöre, es soll und wird blutige Rache werden. Diejenigen, die mich hier haben herbringen lassen, sollen die Köpfe verlieren oder mich ins Zuchthaus bringen lassen, etc."

Nach Trennung der Kranken E. von Frau M. gewann die Geisteskrankheit der ersteren allmälig ihren ursprünglichen Charakter wieder.

Die Wahnideen der Fran M. gründen sich, wie bereits ersichtlich, auf Sinnestäuschungen: sie glaubt, dass Nachts Männer kommen, um Unsittlichkeiten mit ihr zu treiben; vor Allem sei es der Arzt selbst, der sich über ihren "vollen und weissen Körper frent" and sie missbrauche. Auch in der Zeitung habe sie gelesen, dass sie als Dirne feilgeboten werde. Sie hört Ratten über sich, glaubt, mit Steinen beworfen zu werden. Sie hält das Essen für vergiftet; es schmecke und rieche nach "Aas". Sie glaubt, sie werde ron den Behörden verfolgt; man halte sie eingesperrt, da sie um ein Geheimniss wisse, das der Welt verschwiegen bleiben solle. Was dies sei, hat sie dem Arzte nie verrathen, dagegen einmal der Oberin anvertraut: sie sei die Tochter Kaiser Wilhelm I. und der Prinzessin Ulrike. Ihre rechte Mutter bezeichnet sie daher oft als Pflegemutter.

Ihre Sache sei eine Sache des Gerichts; wenn sie herauskomme, werde sie wissen, was sie thue; ihr Mann und sie werden unschuldigerweise verfolgt, an den Bettelstab gebracht, un ihr Vermögen betrogen u. s. w. Die Aerzte sind thre geschworenen Feinde; sie würdigt dieselben keiner Antwort oder aber belegt sie mit den gröbsten Schmähworten. Sie ist in ihren Ausdrücken roh und ausfallend und lässt sich leicht zu aggressiven Acten hinreissen. Unterm 28. December 1896 vermerlkt die Krankengeschichte:

Wirft $A$ bends Tassen und Teller auf den Corridor, schlägt die Thüren zu, schreit mit lauter Stimme, dass es durch das ganze Haus schallt, titulint Arzt und Wärterinnen mit den niedrigsten Schimpfworten, droht, dem Arzt 
den Teller mit Suppe über den Kopf zu giessen, - und dies alles, weil die Suppe angebrannt sein sollte (Geschmackssinntäuschong).

18. März 97. . . . stürzt mit verzerrtem Gesicht auf den Assistenzarzt los, um ihn zu schlagen und sagte u. A.: „Sie wollen mir das Brod wegnehmen, Sie gemeiner Kerl, Sie wollen mich zum Huren verführen, dazu bin ich in diesem Hause“.

Auch das Pflegepersonal, mit dem sie zeitweilig auf vertrauten Fusse steht, besehuldigt sie bei anderer Gelegenheit der ungeheuerlichsten Dinge. Die Zomausbrüche sind plötzlicb, ungestüm und zügellos; das Gesicht wird bleich, die Lippen zucken, die Stimme bebt vor innerer Erregung. In don letzten Jabren gelang es meist, durch weitgehende Berücksichtigung der Individualität den stürmischen Reactionen rorzubeugen.

Das Wahnsystem hat zu durchgreifender Charakterveränderung geführt. Sie meint, einer Welt von persönlichen Feinden gegenüber zu stehen und g]aubt sich in logischer Folgerichtigkeit auch ihrerseits jeder Rü̈cksichtnahme überhoben. Sie ist misstrauisch, menschenfeindlich, unsocial. Sie geht in Erwiderung des an ihr vermeintlich geübten Verfahrens heimliche und krumme Wege bei der Durchführung ihrer Pläne. Sie übertreibt, entstellt die Thatsachen, sucht die Aerzte zu täuschen. So beförderte sie Briefe auf unerlaubtem Wege, durch Einnähen in Kleidungsstücke, an den in der Anstalt befindlichen Mann, entwendete 2 mal der Pflegerin die Schlüssel, um aus der Anstalt ins Freie zu gelangen. Beide Entweichungsversuche (1.Januar und 6. Februar 98) glückten und führten sie in das Haus des Mannes. Ueber den zweiten berichtet die Trrankengeschichte:

Begiebt sich in dic Wohnung ihres Mannes und legt sich daselbst in's Bett. Ihrem Manne erzühlt sie, wie schlecht sie es in der Anstalt habe, und dass der Arzt ihr unsittliche Anträge gemacht habe. Allen diesen Angaben scheint der Mann Glauben zu schenlien. Er fragt den anwesenden Arzt in vorw urfsvollem Tone, wio es damit stehe. Frau M. weigert sich, in die Anstalt zurückzukehren und kann erst unter Zuhülfenabme der Polizei dazu bewegt werden.

Seit diesem Tage haben sich die Ehegatten auf ärtliche Anordnung nicht gesehen. Einige Monate vorher hatte eine Zusammenkunft in der Anstalt stattgefunden; es war die einzige, die aus später zu erläuternden Gründen während des 5jährigen Anstaltsaufenthalts gestattet wurde. Der Mann war inzwischen geheilt und stand kurz vor seiner Entlassung. Die Begrüssung war damals (9. October 97) lant Krankengeschichte eine recht herzliche. Die Frau fragte: "Wie geht es Dir?", worauf Herr M. mit freudestrablender Miene erwiderte: "Gott lob recht gut; ich habe sogar alle Aussicht, bald entlassen zu werden und eine Stellung zu bekommen. Die Herren hier wollen sich für mich verwenden, da ich ja mit mejner Pension nicht ausreichen kann". Frau M.: "Aber G..., lass Dir doch von den Aerzten vichts vormachen, Dich nicht von denselben fangen; das Physicatsattest ist ja doch da, und was geschrieben ist, ist geschrieben". (Sie meint die seiner Zeit ansgesprochene Erklärung, dass der Mann geisteskrank sei, werde aufrecht erhalten bleiben.) 
Sie geht auf keinerlei Gegenvorstellungen ein. „Auch weisst $\mathrm{Du}$ gar vicht G..., was ioh hinter Deinem Rücken gethan, Do kommst hier nicht in Stellung, ich bitte Dich, wir wollen fort nach Berlin". -- "Aber Frau, nun lass mich einmal sprechen. Die Welt ist ja weit, aber wo mir das Brod geboten wird, da 'nehme ich es; ich bin auch der Mann urd muss die Familie ernähren. Werde Du besser, ljebes liind, und dann kommen wir, so Grott will, wieder zusammen". - "Was? Ich besser? bei dem Schweinefrass? Ioh bin nicht krank. Dir haben es die Aerzte wohl so lange eingeredet, dass Du es selbst glaubst". - „Aber Kind, das füble ich doch, dass ich in Vielem zu weit gegangen bin; hier bin ich besser geworden, und es frent mich, dass mich die Aerzte wieder im Stande halten, ein Amt zu bekleiden". - "Die Aerzte? Du woisst dock, das Physicatsattest - wir wollen fort - das andere findet sich schon". - "Nein Frau, beruhige Dich, lass mich nur handeln". Herzliche Verabschiedung.

Ein schriftlicher Verkehr zwischen den Ehegatten wurde in beschränktem Umfange unter ärztlicher Controlle zugelassen. Die Briefe der Kranken sind in geordneter Form, in freundlichen, ja liebevollen Worten abgefasst. Sio zengen von mittlerer Bildung. Regelmässig handeln sie von den wahnhaften Klagen über schlechte Behandlung, Hunger, erlittene Nothzucht, allerhand Ungerechtigkeiten, und schliessen mit der Bitte $\mathrm{nm}$ „Befreiung". Häufigere Nacbrichten werden verlangt, Erkundigungen nach den häuslichen Verhältnissen eingezogen. Gewöhnlich zäht eia langer Wanschzettel eine Reihe mehr oder weniger nützlicher Dinge auf, die der Mann ihr schicken soll. Ich lasse zur Charakterisirung ibres Verhältnisses zu Mann und Sohn einige kurze Proben aus den Briefen folgen, die aus triftigen Gründen nicht zur Absendung gelangten und bei den Acten liegen:

3. September 96. An den Sohn Mein lieber Junge! Ein grosses Bedürfniss ist es für mich, endlich ein Stündchen mit Dir in Gedanken zu plaudern. Vor Allem sage mir, mein Liebling, wie es Dir ergangen ist, bist Du dort gern in Deiner Pension? Bist Du fleissig in der Schule? .... Ueberlassen wir die Rache dem Schicksal, für das uns zugethane bittere Unrecht, es wird eine Stunde der Abrechnugg kommen, Deine Eltern sind unschnldig, lass Dir niemals den Glauben rauben, mein Liebling, Deinen Vater so hoch zu halten, wie er es verdient, und bewahre ihm stets Deine kindliche Liebe; es blieb den Herren nichts anderes übrig, als uns in's Irrenbaus zu stecken, nur so war für sie Rettung möglich ...

2. April 98. Mein lieber Mann und Sohn I.! Gern hörte joh mal atwas won Euch. Nachträglich nimm meinen Glückwunsch zu Deinem Geburtstag .... Hast Du mein Tagebuch schon durchgelesen, dann wirst Du vollständig orientirt sein ... O Ob Dir eine Strafe oder Schuld beigemessen werden darf, hängt ganz von meiner Aussage ab, doch mündlich mehr davon; unmöglich können wir alles frohig hinkehmen, auch des Knaben wegen nicht".

12. Juni 99. (Brief zur heimlichen Beförderung einer Pflegerin übergeben.) „Lieber G . . . . . Vor Allem ist mein Anliegen, dass Du mich hier raus holst; lass Dich doch nicht ron diesem Schweinehund..... ver- 
leiten oder gar bange machen, auch das Gericht schene nicht, sondern schreibe an meine Mutter, dass sie beim Amtsgericht sofort ein Schreiben einreicht..., dass man mich als eine öffentliche Dirne feilbietet, ja sogar mich mit dem Tode bedroht, wenn ich mich dieser schmutzigen Handlung nicht unterwerfe...".

8. Juli 99. ... "Lasse Dich nicht von fremden Menschen beeinflussen, sondern handle selbstständig als Mann".

3. Februar 1901. „Einen Gruss an Mann und Kind; auf baldige Befreiung wartet Deine Frau".

\section{Gutachten.}

Die 45 jährige Lehrersfran M., durch beide Eltern in leichtem Grade nervös belastet, von Jugend auf durch ein absonderliches und leichtsinniges Wesen ausgezeichnet, leidet seit mindestens 6 Jahren an einer Geisteskrankheit, die als chronische Paranoia bezeichnet wird. Sie hat sich allmälig entwickelt unter lebhaften Beziehungswahnideen und Sinnesstörungen auf dem Gebiete des Gehörs, Geschmacks, Geruchs und der Hautempfindung. Auf dieser Grundlage ist ein ausgebreitetes und festgefügtes Wahnsystem entstanden, im Simne der Beeinträchtigung und Verfolgung mit Bevorzugung der geschlechtlichen Sphäre. Daneben bestehen weniger ausgesprochene Grössenwahnvorstellungen. Das so beschaffene Wahngebilde hat naturgemäss zur völligen Umgestaltung der psychischen Persönlichkeit geführt, zu tiefgreifenden Charakter- und Stimmungsveränderungen, die in Misstrauen, Hass, Menschenfeindlichkeit, Unzugänglichkeit, schrofistem Egoismus, maassloser Zornwuth u. dgl. zum Ausdruck gelangen. Diese wieder führten zu Handlungen, die die Kranke in schweren Conflict mit der Aussenwelt bringen. Die Intelligenz schien anfänglich wohl erhalten. Eine methodische Prüfung war durch das ablehnende Verhalten der Kranken allerdings unmöglich gemacht; indessen deutet u. A. das Seltenerwerden der gemütlicben Reactionen, die leichte Abblassung der Simnestäuschungen und der Nachlass der Correspondenz anf beginnende, langsam fortschreitende geistige Schwäche.

Eine Geisteskrankheit derart, welche in keiner Weise von der Regel abweicht, ist erfahrungsgemäss als unheilbar zu betrachten: erstens wegen des charakteristisch-paranoischen Sýmptomenbildes, zweiten wegen der langen Dauer des Bestehens und drittens wegen der qualitativen Unveränderlichkeit der Erscheinungen. Die schwere Geistesstörung wird zeitlebens fortwirken, wenn auch vielleicht die Wahnideen und Sinnestäuschungen durch Zunahme der geistigen Stumpfheit an Intensität noch ein wenig verlieren werden.

Frau M. leidet demach an einer nach Eheschluss über 3 Jahre bestehenden, unheilbaren Geisteskrankheit. 
Hebt die vorliegende Geisteskrankbeit die geistige Gemeinschaft auf?

"Aufhebung der geistigen Gemeinschaft", dieser neugeschaffene Begriff des Bürgerl. Gesetzbuchs, hat bis heute noch keine scharfe Umgrenzung durch die Praxis erfahren können. Die Mannigfaltigkeit geistiger Störungen und ihrer Begleitumstände lässt eine. allgemein gültige Definition des Begriffs äussersit schwierig, wenn nicht unmöglich erscheinen. Auch der vorliegende Fall - schon psychiatrisch in seiner Beziebung zu dem anderen Ehegatten eine interessante Seltenheit - nimmt für die Beurtheilung unserer Frage eine Sonderstellung ein, die es unmöglich macht, ihn unter eine der zur Erklärung des gesetzlichen Begriffes gegebenen Definitionen einzureihen. Trotzdem werden wir auf Grund individueller Betrachtung den Beweis erbringen, dass die geistige Gemeinschaft als aufgehoben zu bezeichnen ist.

Eine Reihe von Momenten, soweit sie die erkrankte Ehegattin selbst :betreffen, scheinen dagegen zu sprechen. Frau M., deren Intelligenz keine groben Defecte aufweist, hat volles Bewusstsein und Verständniss für die Bedentung des ehelichen Verbältnisses. Aus ihren Worten zu schliesser, liegt ihr das Wohl von Maun und Sohn am Herzen. Sie wünscht sehnlichst nach Hause zurückzukehren, schreibt zahlreiche Briefe in Ausdrücken der Zuneigung und in Theilnahme an allen häuslichen Begebenheiten. Allerdings ist nicht zu verkennen, dass das egoistische Bestreben, durch ihren Mamn die Entlassung aus der Anstalt zu erwirken, eine gewisse Rolle spielt. Auch der Hinweis auf die nachweislich wenig liebevolle Behandlung, die sie in der Zeit vor ihrer Aufnahme Mann und Kind angediehen liess, dürfte zu Zweifeln an der Echtheit ihrer jetzigen Gefühle Anlass geben. Ihr Vorleben glich dem einer Prostitnirten utd auch die Geisteskrankheit hat, wie wir sahen, auf indirectem Wege das Gemüthsieben ungünstig beeinflusst.

Indessen, die Charakteranlage, und alles, was dem Ausbruch der Krankheit vorausging, kommt hier nicht in Betracht; auch der durch die Geisteskrankheit veranlasste Niedergang des moralischen Gefühls ist kein so augenfälliger, dass die geistige Gemeinschaft deshalb als aufgehoben zu betrachten wäre. Allzu idealistische Ansprüche an die Qualität der Whe zu stellen, liegt nicht im Sinue des Gesetzes. Bemerkenswerth ist, dass die Wahnideen durchaus nicht, wie oft in ähnlichen Fällen, einen dem Manne feindlichen Charakter tragen. Das Gegentheil ist der Fall: auch der Mann erscheint ihr als ein unschuldiges Opfer geheimer Intriguen, er leidet in ihrer Vorstellung mit ihr unter den gleichen 
Verfolgungen der Behörden. Sie kämpfte für seine „Befreinng" aus der Anstalt mit gleicher Wärme wie für die eigene.

Wir nehmen also an, dass Bewusstsein und Wille, die ehclichen Interessen zu fördern, in genügendem Maasse vorhanden sind, und fragen weiter, ob diesem Willen die Fähigkeit zur Bethätigung entspricht.

Frau M. ist gemeingefährlich. Schwere Störungen der öfentlichen Ordnung, die seiner Zeit die Aufnahme in die Irrenanstalt veranlassten, wären auch heute noch als Folgewirkungen ihrer Beeinträchtigungsideen unausbleiblich. Eine voraussichtlich dauernde Anstaltsbedürftigkeit liegt vor; indessen schliesst eine solche keineswegs unbedingt das Vorhandensein der geistigen Gemeinschaft aus, wie wir in Uebereinstimmung mit den meisten Gutachtern ausdrücklich betonen wollen.

Selbt gemeingefährliche Geisteskranke sind sehr wohl in der Lage, durch Empfang von Besuchen die Familienbeziehungen in der Anstalt fortzusetzen, am Wobl und Wehe der Angehörigen theil zu nehmen. Wenn mündlicher Verkehr aus irgend einem Grunde unstatthaft, tritt der schriftliche an dessen Stelle. So lässt sich trotz räumlicher Trennung das geistige Band der ehelichen Gemeinschaft aufrecht erhalten.

Die äusseren Bedingungen dazu sind auch in unserem Falle auf Seiten der Kranken gegeben; die ärztliche Rücksicht auf den gesunden Ehegatten dagegen verbietet jede intime Verkehrsbeziehung; (selbst die schriftliche, sofern es sich nicht um gleichgültige Dinge handelt). Wir werden zeigen, dass eine solche Verkehrsbeziehung jenen gesundheitlich schädigt, indem sie ihn der sicheren Gefahr überantwortet, ebenfalls wieder geistig zu erkranken.

Es bedarf kaum der Erwähnung, dass die Berücksichtigung des gesunden Ebegatten bei der vorliegenden Frage gesetzlich gereehtfertigt ist. Eine kürzlich ergangene Landgerichtsentscheidung ${ }^{1}$ ) (bestätigt durch das Hanseatische Oberlandesger., II. Civilsenat, Erkenntaiss vomı 22. I. 01. Bf. Il. 293/1900) geht ausdrücklich davon aus, "dass man nicht einseitig den Zustand des Geisteskranken, sondern auch die Lage des gesunden Ehegatten, zu dessen Gunsten doch das Gesetz gemacht sei, in Betracht ziehen müsse."

Der pens. Lehrer M. ist seit 1884 in zweiter Ehe mit der Kranken verheirathet. 1886 fiel seinen Kollegen zuerst eine geistige Veränderung an ihm auf. Diese steigerte sich in Laufe, der Jahre, verlief

1) S. auch Psych. Wochenschr. 1901. No. 8. 
unter den gleichen Erscheinungen der sogenannten Paranoia, wie die Krankheit der Frat, führte zu Beleidigungen und Insulten seiner Vorgesetzten uud machte, wie erwähnt, am 29. October 1895 auf polizeiliche Anordnung die Aufnahine in die Irrenanstalt erforderlich.

Bei genauer Prüfung aller einschlägigen Verhältnisse ergab sich schon vor der Aufnahme der Frau, dass die Gleichzeitigkeit der Erkrankungen beider Ehegatten nicht auf Zufall beruhen konnte; es liess sich ferner ausschliessen, dass etwa gleiche Schädlichkeiten zwei von einander unabhängige Krankheiten veranlasst hätten; vielmehr wurde schon damals festgestellt, dass beide Krankheitsfälle in einem nahen, inneren Zusammenhange standen. Der eine war die Folge des anderen. Eine offenbare Krankheits ïbertragung hatte stattgefunden; es handelt sich um einen jener seltenen, aber wissenschaftlich wohlbekannten Fälle von Folie à deux (Folie communiqué, inducirtem Irresein).

Inducirtes Irresein kommt dadurch zu Stande, dass durch Ueberpflanzung eines Wahnsystems auf ein zweites, bis dahin gesundes Individuum, das mit dem Ersterkrankten in inniger Gemeinschaft Jebt, eine Geisteskraukheit gleichartigen Charakters hervorgerufen wird.

Dieser Thatbestand ist in unserem Falle zu erweisen, und zwar in dem Sinne, dass die Ehefrau den primär-erkrankten, der Ehemann den inficirten Theil darstellt. Nur bei einem solchen Beziehungsverhältniss ist der weitere Schluss gerechtfertigt, dass die Fortsetzung der intimen Ehegemeinschaft den zur Zeit gesunden Ehemann in seinen Lebensinteressen schüdigt.

Gesetzt den Fall, es wäre umgekehrt, d. h. der Ehemann der active, krank machende Theil - wie seine 1/2 Jahr früher erfolgte Aufnahme in die Anstalt vermuthen liesse und wie es anfünglich in der That auch ärztlicherseits angenommen wurde - so würden wir in der Beantwortung der forensischen Frage liaum zu gleichem Resultat gelangen; denn das Zusammenleben eines genesenen, früher primär erkrankten Ehemannes mit einer ungeheilten, "inficirten" Frau gäbe keine zwingende Veranlassumg; eine Wiedererkrankung des ersteren befürchten zıı müssen.

Dass der Primär-Erkrankte gesund, der inducirte Theil dagegen unheilbar wird, wäre kein hypothetiseher Fall, sondern ein in der Literatur bekanntes; allerdings sehr seltenes Ereigniss. Das Umgekehrte, wie es bei uns der Fall ist, entspricht der Regel.

Es war in die Augen springend, wie nach erfolgter Trennung der Ehegatten bei dem inficirten Ehemanne alle wahnhaften Aeusserungen wie mit einem Schlage verschwanden, während die Krankheit der Frau bis heute unverändert fortbesteht. So aber entspricht es bei diesem 
Beziehungsverhältniss aller Erfahrung. Weun der Mann trotzdem ca. 2 Jahre in der Anstalt verblieb, so geschah dies aus anderen, nicht hierher gehörenden Gründen. Auch der Beginn der Erkrankungen lässt keinen Zweifel über die Priorität. Charakter und Lebensführung der Frau zeigten von Jugend auf abnorme Formen und krankhafte Neigungen, während der Mann bei normalem Verbalten seinem Lebrerberuf oblag, bis sich bald nach der zweiten Heirat die ersten Anzeichen geistiger Störung bemerkbar machten. Die vorurtheilsfreie Schilderung der Angehörigen ergab, dass er vom ersten Tage der Ehe an, dem übermächtigen Einflusse der Frau verfiel und sich willenlos ihrem Despotismus beugte. Sie leitete ihn, und sie war ihm zweifellos auch intellectuell überlegen. Wer ihn kennt, weiss, wie lenksam, eindruckfähig, wachsweich dieser Mann ist. Seine geistigen, insbesondere kritischen Fähigkeiten sind umbedeutend. Ausserdem kommt als wich. tiges Moment hinzu, dass er von Hause aus zu Geisteskrankheit veranlagt ist. Er stammt - wie dies fast inmer bei inducirtem Irresein der Fall ist - aus erblich stark belasteter Familie; der Vater war Trinker, 2 Schwestern waren geisteskrank, 2 Schwesterkinder sind schwachsinnig.

Auf diesem Untergrunde fanden die Wabuideen der Ehefrau begreiflicherweise einen günstigen Nährboden. Aber nicht nur die Uebermacht der Persönlichkeit des einen, die Widerstandslosigkeit des anderen Theils, sondern anch die Form der primären Geisteskrankheit ist als wirksamer Faktor in Rechnung zu ziehen. Sie betrifit, wie wir zeigúen, weniger das Gefühls-, als das Vorstellungsleben, sie gehört in die Gruppe der Paranoia, und nur solche Krankheiten sind es, die erfahrungsgemäss und logischer Weise unter günstigen Umständen inducirende Kraft entfalten.

Als Schlussstein tritt hinzu, dass die Wahnideen der Ehef'au, besomnen und in fester Form vorgerragen, den Bereich des Möglichen irn Anfang kaum überschritten, somit den Stempel einer gewissen G]aubwürdigkeit trugen, und endlich dem Inbalte nach sich fast ausschliesslich in der Ideen- und Interessensphäre des ll annes bewegten. Wie aus den zahlreichen, oben mitgetheilten Aeusserungen hervorseht, war und blieb der Mann und sein Berufskreis in den Wahn der Frau stets mit einbezogen.

Klar und unzweideutig sprecben für unsere Annahme die eigenen Worte des geheilten Mannes; er äusiserte schon am 10. October 97 dem Arzte gegenüber: "Leider hat sie mich den ganzen Tag mit ihren Vorstellungen gequält, beständig an mir gebohrt und gehetzt, sonst 
wäre es nie soweit gekommen; zuletzt glaubte ich sogar an das Unmögliche."

Die Identität der gemeinsamen Wahnvorstellungen führte zu gleichen Conflicten mit der Aussenwelt, den gleichen Persönlichkeiten gegenüber. Die Frau stachelte den Mann zu Gewalthätigkeiten, schob ihn vor und blieb daher lange Zeit als die eigentliche causa movens verborgen. Auf diese Weise erklärt es sich, dass die Krankheit der Frau trotz ihres früheren Beginns später als die des Mannes in die öfentliche Erscheinung trat und später zur Aufnahme in die Irrenanstalt führte.

Wie nachhaltig der Einfluss jener Uebermacht und wie zwingend daber die absolute, dauernde Trennung der Ehegatten geboten ist, lehren die Erfahrungen der Folgezeit.

Wir zeigten an dem ausführlich geschilderten Beispiel der mitkranken Frau E., dass die Krankheit der Frau M. im Laufe der Jahre an Inductionskraft nichts verloren hat. Vor allem aber sei betont, dass, so oft eine unbeabsichtigte neue Berïhrung mit dem Ehemanne erfolgte, regelmässig eine Verschlechterung seines geistigen Befindens eintrat. Die Krankengeschichte des Mannes erhält darüber unter dem 13. Februar 1898 folgenden Nachtrag, dem ein Bericht der Schwester zu Grunde liegt:

"So lange der p. M. von dem Einflusse der Ehefrau befreit war, hat er sich sehr gut befunden, wach der ersten Entweichung der Frau jedoch trat wieder eine Verschlimmerung ein, die nach der zweiten Entweichung sich noch gesteigert hat." Und als bei letzterer Gelegenheit der Arzt die Kranke im Hause aufsuchte, liess sich feststellen, dass der M. sofort wieder den wahnhaften Anklagen der Ehefran Glauben schenkte, selbst den absurdesten, die darin bestanden, dass der anwesende Arzt der Kranken unsittliche Anträge gemacht hätte.

Mühsam und allmälig hat sich M. im Laufe der Zeit von dem dïmonischen Einfluss seiner Frau losgemacht, Es kostete ibn, wie er kürzlich in einer Unterredung mit dem Arzte sagte, "schwere Selbstüberwindung, um sie ganz zu vergessen." Er ist gesund geblieben, seine Entmündigung ist aufgehoben, er bekleidet seit seiner Entlassung aus der Anstalt eine Stelle als Schreiber beim Bauamt.

Das Ziel ärztlicher Bemühungen, zwei sich pathologisch anziehende, sich social abstossende Elemente äusserlich und innerlich von einander zu trennen, ist somit erreicht. Die Ehescheidung würde an dem bestehenden Zustande nichts ändern, sondern einzig und allein die staatliche Anerkennung einer durch die Macht der Verhältnisse vollzogenen Trennung bedeuten. Die Ehescheidung ist erwünscht; denn sie 
würde besser, als dies bisher durch den Arzt geschehen konnte, das Nichtzustandekommen einer Wiedervereinigung gewährleisten. Dio Ehescheidung ist daher nicht nur als zulässig, sondern - gewiss ein seltener Fall! - als geboten zu erachten. Die geistige Gemeinschaft ist für die juristische Beurtheilung als aufgehoben zu betrachten, für jetzt und alle Zukunft, weil sie aus ärztlichen Gründen zwingendster Natur dawernd aufzuheben ist. Käme sie je wieder zu Stande, so würde der heute gesunde Ehegatte zweifellos wieder in Geisteskrankheit verfallen. Ja selbst bei dem Sohu ist die gleiclet Gefahr nicht ansgeschlossen; er zeigte sich, wo oben erwähnt, bereits eimmal den Beeinflussungen der Mutter so stark unterworfen, dass auch an ihm vorübergehend eine Induction befürch tet werden musste.

Ich gebe mein Gutachten dahin ab, dass in Sachon der beklagten Frau M. die Bedingungen des $\$ 1569$ B. G. B. erfüllt sind.

Die Ehe wurde geschieden. Dex Richter bekante sich zu der neuen, psychiatrisch geforderten Auslegung des Gesetzes. Die Bosonderheit leuchtet ein: In der Norm kommt ausschliesslich die Qualification der in Rede stehenden, absolut betrachteten Geisteskrankheit in Frage; diese gab - nach unserer vielleicht etwas strengen Auffassung - zur Anwendung des Gesetzes keine Handhabe. Bedeutungsvoll wurde sie allein durch ihre relative Ligenart. Eine für vorliegenden Zweck indifferente Psychose gewann ihre Qualification dadurch, dass sie auf den ebenfalls (qualificirten Ehegatten inducirende Sraft ausübte. Der Schwerpunkt liegt nicht in dem diagnostischen Zustandsbilde, sondern in der prognostisch maassgeblichen Wirkungsausserung, deren Eintritt mit Bestimmtheit gerale an die ebelicho Wiedervereinigung geknipft ist.

In klinischer Beziehung hält sich der Fall in den Grenzen des von Schön $f^{1} \mathrm{~d}^{1}$ ) am engsten umschriebenen Bilkes. Der paranoische Charakter, die Gleichartigkeit der Symptome, das geistige Abläu gigkeitsverhältniss zwiscben den Betheiligton, die psychotische Disposition u. s. w. sind genügend scharf betont worden. Xur in einem Punkte scheint den Forderungen Schönfeid's nicht genügt zu sein; er stellt als Regel auf, dass die secundäre Erkranknug auch nach erfolgter Trennung "ihren typischen Verlaut" nimmt. Das war nicht der Fall. Der inducirte Ehemann ärnserte nach der Aufnahme weder Wahnideen noch Sinnestäusehungen; auch aus seinen Handlungen

1) Schönfeld, Ceber das inducirte Irresein. Dieses Archiv Bd. XXVI. 
ging nichts' Auffälliges mehr hervor. Hingegen liess allerdings das Benehmen noch viele Monate auf krankhafte Ideengänge schliessen. M. war einsilbig, wich anzüglichen Fragen aus, trug Misstrauen zur Schau, und erst im zweiten Jahr bekannte er Krankheitseinsicht. Ein 2jähriger Verbleib in der Anstalt war angezeigt, da lange Zeit der Verdacht der Dissimulation 'begründet war.

Zur völligen Klarlegung der Verhältnisse wäre es angebracht, das im Gutachten skizzirte Krankheitsbild des Ehemanns zu vervollständigen. Doch davon kömnen wir an dieser Stelle Abstand nehmen, da derselbe Fall schon ausführlich von Riedel1) in der Literatur behandelt ist, Bereits Riedel stellte das Vorliegen einer klassischen Folie à deux fest, nur dass er das umgekehrte Causalitätsverhältniss zwischen den Psychosen aunimmt. Für ihn ist der M an der Ersterkrankte. Dieser aufünglich allgemein getheilte Irrthum ist begreiflich. 1896, als Riedels Arbeit erschien, waren beide Ehegatten noch in der Anstalt, und zwar die Frau erst kurze Zeit, da ihre Psychose aus genannten Gründen später, als die des Mannes, die öffentliche Aufmerksamkeit auf sich gezogen hatte. Erst die rasch und vollständig eintretende Heilung des Mannes liess die Vermuthung auftauchen, dass er der secundür Erkrankte sei. Diese Vermuthung wurde zur Wahrscheinlichkeit, als Genaueres über die Vorgeschichte der Frau bekannt wurde, und fand ihre Bestätigung durch weitere sorgfältige Nachforschungen und Beobachtungen, sowie durch den späteren Gesammtkrankheitsverlauf heider Ehegatten.

Eine Erscheinung, die uns ebenfalls zur Stütze des dargelegten Standpunktes diente, war die vorïbergehende Uebertragung des Wabnsystems der Frau M. auf eine andere Kranke innerhalb der Anstalt. Hier vollzog sich unter den Augen des Arztes ein ganz ăhnlicher Vorgang, wie draussen am Mann und andeutungsweise am Sohn. Es war eine dritte Induction, doch mit dem Lnterschiede, dass es sich nicht um Einpflanzung einer neuen Geisteskrankheit handelte, sondern nur um Uebermittelung charakteristischer Wahnvorstellungen auf eine schon kranke Person.'

In der Literatur des inducirten Irreseins fand ich keinen Fall, der in so demonstrativer Weise die psychische "Infectionskraft" des activen Theils auch nachträglich noch zum Ausdruck brachte. Bei anderen Kranken, nicht aus der Gruppe der communicirten Psychosen, wird vereinzelt über ähnliche Beeinflussungen berichtet. Die Franzosen nennen es "Folie transformeé". Schönfeld²), der diese Erscheinung

1) Riedel, Vierteljahrsschr. f. ger. Med. 3. Folge. XIV. 2.

2) l. c. 
als sehr seltenes Kreigniss bezeichnet, citirt diesbezügliche Mittheilungen von Pronier und von Kiernan. In allerjüngster Zeit hat $\mathrm{Näcke}^{1}$ ) einen "Beitrag zur gegenseitigen Beeinflussung von Geisteskranken" geliefert, in dem er ebenfalls die ausserordentliche Seltenheit solcher Wahmehmungen betont. Er nennt Fälle von Burzio, Norel und van Deventer, aber fügt hinzu, dass bei allen diesen keine Assimilirung fremder Wahnideen, sondern nur eine flüchtige Hinnahme, eine Art "Gedankeninfection" stattfand. Schönfeld, wie Näcke, sehen ziemlich übereinstimmend die Ursache für das seltene Vorkommen von Inductionserscheinungen in Irrenanstalten einmal in der starken Inanspruchnahme der Kranken durch die eigenen inneren Vorgänge und zweitens in der Oberflächlichkeit der gemüthlichen Beziehungen der Kranken zu einander.

In unserem Falle wird man sich der Einsicht nicht verschliessen können, dass die wirkungsvolle Activität der primären Kranken eine Hauptrolle gespielt hat. Die Inducirte ist keineswegs als sehr suggestibel zu bezeichnen. Das Zusammenleben war durchaus kein besonders inniges. Die beiden Kranken bewohnten nicht einmal ein gemeinsames, sondem 2 aneinanderstossende Zimmer. Dagegen waren die Verhältnisse bei dem empfangenden Theil insoferı günstige, als die Psychose in keimender Wahnentwicklung stand und ähnlichen Tendenzen, wie die andere sie bot, zuneigte. Sie äusserte sich seit ihrem $3 / 4$ jällrigen Bestehen in der Form einer Hallucinose, welche chronisch zu werden und das paranoische Stadium zu bilden begann. Ob Sinnestäuschungen übernommen wurden, war nicht sicher festzustellen, im Wesentlichen handelte es sich um fremden Neuerwerb von Wahnideen. Bis heute siud 10 Monate seit erfolgter Trennung vergangen. Der inducirte Wahn ist dauernd verschwunden: die Systematisirung des primären vollzieht sich im Rahmen des ursprünglichen Bildes.

1) Neurol. Centralbl. 1901. No. 14. 